\title{
Weight Optimization of Optimal Control Influenza Model Using Artificial Bee Colony
}

\author{
Dinita Rahmalia and Teguh Herlambang
}

\begin{abstract}
Influenza is disease which can be contagious through contact with infected individual. There are two types of control strategies to bound the spread of disease: prevention action for controlling susceptible and treatment for controlling infected. Optimal control is used for minimizing the number of infected individual, the cost of prevention action and the cost of treatment. Due to the cost of objective function depends on weight, in this research we will apply Artificial Bee Colony algorithm to optimize weight minimizing cost of objective function. The simulations show that the number of infected with control is lower than without control. Furthermore, we also obtain optimal weight related to cost of prevention action and treatment.

Index Terms-Artificial Bee colony, influenza model, optimal control.
\end{abstract}

\section{INTRODUCTION}

$\mathbf{N}$ OWADAYS, there are many contagious diseases which have been found. In contagious disease, there are susceptible individuals, infected individuals, and recovered individuals. Susceptible individual can be infected individual after making contact with infected individual based on disease transmission rate. Then, infected individual becomes recovered individual when the symptoms of disease have lost. But, recovered individual can return to susceptible when the immunity has lost.

Influenza, commonly known as "the flu", is an infectious disease caused by influenza virus. The most common symptoms are high fever, runny nose, sore throat, muscle pains, headache, coughing, and feeling tired. Usually, this virus spread through the air from coughs or sneezes. It can also spread by touching surfaces contaminated by virus [1].

The purpose of epidemics modeling is to provide policies designed to control the spread of disease [2]. There are two types of control strategies to bound the spread of disease: prevention action for controlling susceptible and treatment for controlling infected. Preventive action may be good sanitation and healthy food. Treatment may be drug for healing the disease. Optimal control is used for minimizing the number of infected individuals, the cost of prevention actions and the cost of treatments [3].

In the earlier research from Michalewicz, by heuristic optimization like Genetic Algorithm (GA), we can determine optimal control minimizing objective function based on natural selection of chromosomes [4]. The Artificial Bee Colony

Manuscript received October 11, 2017; accepted January 23, 2018

D. Rahmalia is with the Mathematics Department, Universitas Islam Darul Ulum, Lamongan, Indonesia. E-mail: dinitarahma lia@gmail. com

T. Herlambang is with the Information System Department, Universitas Nahdlatul Ulama, Surabaya, Indonesia. E-mail: teguh@unusa.ac.id
(ABC) was introduced by Karaboga and Akay in 2009. ABC is the optimization method which is inspired by the behavior of bee colony. In the $\mathrm{ABC}$ algorithm, there are three types of bees, namely employed bees, onlooker bees, and scout bees. For every food source, there is only an employed bee. The position of food source represents possible solution of the optimization problem and the amount of nectar represents the fitness of solution [5].

In optimal control problem, weight selection is applied by trial and error [6]. Due to the cost of objective function that depends on weight, in this research we will apply $\mathrm{ABC}$ algorithm to optimize weight minimizing cost of objective function. In previous research, Ant Colony Optimization (ACO) has been applied on contagious disease [7]. The weight used are related to the number of infected individuals, cost of prevention actions and treatment for controlling the number of susceptible individuals and infected individuals, respectively.

Simulations have been applied and we can obtain comparison the number of individual with and without control. In addition, we also obtain optimal weight related to cost of preventive actions and treatment for controlling the number of susceptible individuals and infected individuals, respectively.

\section{Artificial BeE Colony}

\section{A. The Behavior of Bee}

There are three groups in the $\mathrm{ABC}$ : employed bees, onlooker bees, and scouts. For every food source, there is only an employed bee. The position of food source represents possible solution of the optimization problem and the amount of nectar represents the fitness of solution.

The number of employed bees or the onlooker bees is equal to the number of individual in the population. The employed bees produce the position of food source depending on the information and test the amount of nectar.

After all employed bees complete the search process, they share the position of food source and the nectar information with the onlooker bees by dance area. The onlooker bees test nectar information and choose a position of food source with the best probability.

The position of food source of which the nectar is abandoned by the bees is replaced with the new position of food source by the scout bees.

\section{B. Algorithm of $A B C$}

The algorithm of $\mathrm{ABC}$ is as follows [5], [8]. Given objective function $f: X \subseteq \mathbb{R}^{D} \rightarrow \mathbb{R}$ where $D$ is the dimension of the search space. In the initialization step, generate initial solutions 
$x_{i j}, i=1, \ldots, S N, j=1, \ldots, D$ randomly and evaluate the fitness $f\left(x_{i j}\right)$, for $i=1, \ldots, S N, j=1, \ldots, D$.

We repeat execution of the following steps:

1) Employed Bees Step

In the employed bees step, each employed bee generates solution as follows:

$$
v_{i j}=\left\{\begin{aligned}
x_{i j}+\phi_{i j}\left(x_{i j}-x_{k j}\right), i \neq k, & \text { if } r_{i j}<M R \\
x_{i j}, & \text { otherwise }
\end{aligned}\right.
$$

Then we evaluate $f\left(v_{i j}\right)$, for $i=1, \ldots, S N, j=1, \ldots, D$. If new solution $v_{i j}$ is better than $x_{i j}$, replace $x_{i j}$ with $v_{i j}$.

2) Onlooker Bees Step

Onlooker bees select one of solutions based on probability:

$$
p_{i}=\frac{\text { fitness }_{i}}{\sum_{n=1}^{S N} \text { fitness }_{n}}
$$

where in the minimization problem, the fitness can be obtained from:

$$
\text { fitness }_{i}=\left\{\begin{aligned}
\frac{1}{1+f\left(x_{i}\right)}, & \text { if } f\left(x_{i}\right) \geq 0 \\
1+\left|f\left(x_{i}\right)\right|, & \text { if } f\left(x_{i}\right)<0
\end{aligned}\right.
$$

3) Scout Bees Step

Determine the abandoned solution for the scout and replace it with:

$$
x_{i j}=x_{j}^{\min }+\theta_{i j}\left(x_{j}^{\max }-x_{j}^{\min }\right)
$$

where $\theta_{i j} \sim U(0,1)$ then evaluate $f\left(x_{i j}\right)$, for $i=$ $1, \ldots, S N, j=1, \ldots, D$.

4) Keep the Best Solution

\section{Optimal CONTROL OF SIR EPIDEMic MODEL}

We can classify influenza as SIR epidemic model [3]. At the SIR epidemic model, there are three compartments of individuals: susceptible, infected, and recovered. Susceptible individual can become an infected individual after making a contact with infected individual based on disease transmission rate. Infected individual can become a recovered individual when the symptom of disease have lost based on recovery rate. Recovered individual can become a susceptible individual when the immunity has lost based on the lost immunity rate.

At the susceptible compartment, recruitment rate and the lost immunity rate can increase the number of susceptible individuals. However, disease transmission rate and natural death rate can decrease the number of susceptible individuals.

At the infected compartment, disease transmission rate can increase the number of infected individuals. However, natural death rate, death by disease rate, and recovery rate can decrease the number of infected individuals.

At the recovered compartment, recovery rate can increase the number of recovered individuals. However, natural death rate and the lost immunity rate can decrease the number of recovered individuals.

The compartment of SIR epidemic model can be seen on Fig. 1.

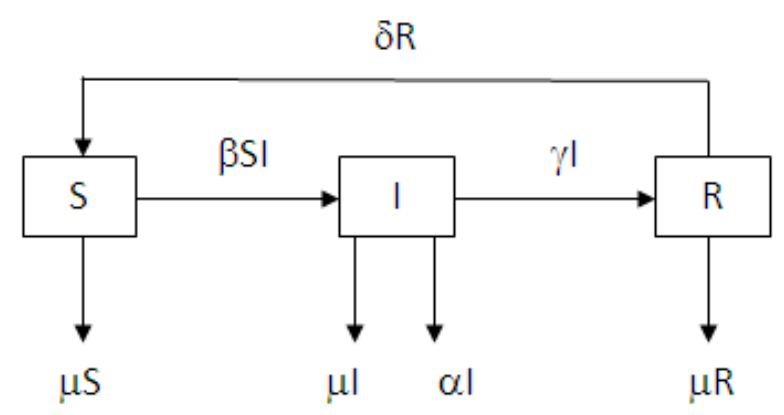

Fig. 1. Compartment Diagram of SIR Epidemic Model.

\section{A. Mathematical Model}

The mathematical model of optimal control influenza model can be described as follows:

$$
\begin{aligned}
\dot{S} & =\Lambda-\beta S I-\mu S+\delta R-u_{1} S \\
\dot{I} & =\beta S I-\alpha I-\mu I-\gamma I-u_{2} I \\
\dot{R} & =\gamma I-\mu R-\delta R+u_{1} S+u_{2} I
\end{aligned}
$$

where $S(0) \geq 0, I(0) \geq 0, R(0) \geq 0$ with the parameters: recruitment rate $\Lambda$, disease transmission rate $\beta$, natural death rate $\mu$, death by disease rate $\alpha$, recovery rate $\gamma$, and the lost immunity rate $\delta$. In addition, there are control function of susceptible prevented $u_{1}$ and control function of infected treated $u_{2}$.

The objective function which will be minimized is:

$$
J\left(u_{1}, u_{2}\right)=\int_{0}^{T}\left(A_{1} I+A_{2} \frac{u_{1}^{2}}{2}+A_{3} \frac{u_{2}^{2}}{2}\right) d t
$$

with weight $A_{1}>0, A_{2}>0, A_{3}>0$. From the model, we want to minimize the number of infected individuals, the cost of preventive actions, and the cost of treatments. The goal is finding $u_{1}^{*}, u_{2}^{*}$ such that

$$
J\left(u_{1}^{*}, u_{2}^{*}\right)=\min \left\{J\left(u_{1}, u_{2}\right):\left(u_{1}, u_{2}\right) \in U\right\}
$$

where $U$ is the set of admissible controls defined by: $U=$ $\left\{\left(u_{1}, u_{2}\right): 0<u_{1}<1,0<u_{2}<1\right\}$ and $u_{1}, u_{2}$ are Lebesgue measurable.

\section{B. Pontryagin's Maximum Principle}

If $u_{1}^{*}, u_{2}^{*}$ is an optimal control corresponding state system, there exist adjoint variables $\left(\lambda_{S}, \lambda_{I}, \lambda_{R}\right)$ which satisfy the following [3]:

$$
\begin{aligned}
& \dot{\lambda}_{S}=-\frac{\partial H}{\partial S}=-\lambda_{S}\left(-\beta I-\mu-u_{1}\right)-\lambda_{I} \beta I-\lambda_{R} u_{1} \\
& \dot{\lambda}_{I}=-\frac{\partial H}{\partial I}=-A_{1}-\lambda_{S}(-\beta S)-\lambda_{I}\left(\beta S-\alpha-\mu-\gamma-u_{2}\right)-\lambda_{R}\left(\gamma+u_{2}\right) \\
& \dot{\lambda}_{R}=-\frac{\partial H}{\partial R}=-\lambda_{S}(\delta)-\lambda_{R}(-\mu-\delta)
\end{aligned}
$$


where $\lambda_{S}(T)=\lambda_{I}(T)=\lambda_{R}(T)=0$, and the Hamiltonian is:

$$
\begin{aligned}
H=\left(A_{1} I+A_{2} \frac{u_{1}^{2}}{2}+A_{3} \frac{u_{2}^{2}}{2}\right)+ & \\
& \left(\begin{array}{lll}
\lambda_{S} & \lambda_{I} & \lambda_{R}
\end{array}\right)\left(\begin{array}{c}
\Lambda-\beta S I-\mu S+\delta R-u_{1} S \\
\beta S I-\alpha I-\mu I-\gamma I-u_{2} I \\
\gamma I-\mu R-\delta R+u_{1} S+u_{2} I
\end{array}\right)
\end{aligned}
$$

Furthermore, we can find optimal control $u_{1}^{*}, u_{2}^{*}$ :

$$
\begin{aligned}
\frac{\partial H}{\partial u_{1}} & =0 \\
A_{2} u_{1}-\lambda_{S} S+\lambda_{R} S & =0 \\
u_{1} & =\min \left(1, \max \left(0, \frac{\left(\lambda_{S}-\lambda_{R}\right) S}{A_{2}}\right)\right) \\
\frac{\partial H}{\partial u_{2}} & =0 \\
A_{3} u_{2}-\lambda_{I} I+\lambda_{R} I & =0 \\
u_{2} & =\min \left(1, \max \left(0, \frac{\left(\lambda_{I}-\lambda_{R}\right) I}{A_{3}}\right)\right)
\end{aligned}
$$

\section{Forward Backward Difference}

To solve optimal control SIR epidemic model, we need a discretization process using forward backward difference approximation where initial conditions for the state equations and terminal conditions for the adjoint equations.

The discretization of forward backward difference can be described as follows: Set $S_{0}, I_{0}, R_{0}, \lambda_{S, T}=\lambda_{I, T}=\lambda_{R, T}=0$, $u_{1,0}=u_{2,0}=0$.

$$
\begin{aligned}
& \frac{S_{k+1}-S_{k}}{h}=\Lambda-\beta S_{k+1} I_{k}-\mu S_{k+1}+\delta R_{k}-u_{1, k} S_{k+1} \\
& \frac{I_{k+1}-I_{k}}{h}=\beta S_{k+1} I_{k+1}-\alpha I_{k+1}-\mu I_{k+1}-\gamma I_{k+1}-u_{2, k} I_{k+1} \\
& \frac{R_{k+1}-R_{k}}{h}=\gamma I_{k+1}-\mu R_{k+1}-\delta R_{k+1}+u_{1, k} S_{k+1}+u_{2, k} I_{k+1} \\
& \frac{\lambda_{S, T-k}-\lambda_{S, T-k-1}}{h}=-\lambda_{S, T-k-1}\left(-\beta I_{k+1}-\mu-u_{1, k}\right)- \\
& \quad \lambda_{I, T-k}\left(\beta I_{k+1}\right)-\lambda_{R, T-k}\left(u_{1, k}\right) \\
& \frac{\lambda_{I, T-k}-\lambda_{I, T-k-1}}{h}=-A_{1}-\lambda_{S, T-k-1}\left(-\beta S_{k+1}\right)- \\
& \quad \lambda_{I, T-k-1}\left(\beta S_{k+1}-\alpha-\mu-\gamma-u_{2, k}\right)-\lambda_{R, T-k}\left(\gamma+u_{2, k}\right) \\
& \frac{\lambda_{R, T-k}-\lambda_{R, T-k-1}}{h}=-\lambda_{S, T-k-1}(\delta)-\lambda_{R, T-k-1}(-\mu-\delta) \\
& A_{2} u_{1}-\lambda_{S} S+\lambda_{R} S=0 \\
& A_{3} u_{2}-\lambda_{I} I+\lambda_{R} I=0
\end{aligned}
$$

\section{Overall Algorithm}

In this algorithm, the weight used are $A_{1}, A_{2}, A_{3}$ related to the number of infected individuals, cost of preventive actions and treatment for controlling the number of susceptible individuals and infected individuals, respectively.

The algorithm of SIR function with the parameter $A_{1}, A_{2}$ and $A_{3}$ can be constructed as follows :

$$
\operatorname{SIR}\left(A_{1}, A_{2}, A_{3}\right)
$$

Set $S_{0}, I_{0}, R_{0}, \lambda_{S, T}=\lambda_{I, T}=\lambda_{R, T}=0, u_{1,0}=u_{2,0}=0$

For $k=0, \ldots, T-1$ we compute

$$
\begin{aligned}
& S_{k+1}=\frac{h \Lambda+h \delta R_{k}+S_{k}}{1+h \mu+h \beta I_{k}+h u_{1, k}} \\
& I_{k+1}=\frac{I_{k}}{1-h \beta S_{k+1}+h(\alpha+\mu+\gamma)+h u_{2, k}} \\
& R_{k+1}=\frac{h \gamma I_{k+1}+h u_{1, k} S_{k+1}+h u_{2, k} I_{k+1}+R_{k}}{1+h \mu+h \delta} \\
& \lambda_{S, T-k-1}=\frac{\lambda_{S, T-k}+h \lambda_{I, T-k}\left(\beta I_{k+1}\right)+h \lambda_{R, T-k}\left(u_{1, k}\right)}{h\left(\beta I_{k+1}+\mu+u_{1, k}\right)+1} \\
& \lambda_{I, T-k-1}=\frac{h A_{1}+\beta S_{k+1}\left(-h \lambda_{S, T-k-1}\right)+h \lambda_{R, T-k}\left(\gamma+u_{2, k}\right)+\lambda_{I, T-k}}{1+h\left(-\beta S_{k+1}+\alpha+\mu+\gamma+u_{2, k}\right)} \\
& \left.\left.\lambda_{R, T-k-1}=\frac{h \lambda_{S, T-k-1}(\delta)+\lambda_{R, T-k}}{h(\mu+\delta)+1}\right)\right) \\
& u_{1, k+1}=\min \left(1, \max \left(0, \frac{\left(\lambda_{S, T-k-1}-\lambda_{R, T-k-1}\right) S_{k+1}}{A_{2}}\right)\right) \\
& u_{2, k+1}=\min \left(1, \max \left(0, \frac{\left(\lambda_{I, T-k-1}-\lambda_{R, T-k-1}\right) I_{k+1}}{A_{3}}\right)\right)
\end{aligned}
$$

Then we calculate the objective function as fitness function [9]:

$$
J\left(u_{1}, u_{2}\right)=\sum_{k=0}^{T-1}\left(A_{1} I_{k}+A_{2} \frac{u_{1, k}^{2}}{2}+A_{3} \frac{u_{2, k}^{2}}{2}\right)
$$

The overall algorithm combining ABC and optimal control influenza model is as follows:

(1) In the initialization step, generate initial solutions $A_{i}=$ $\left(A_{1_{i}}, A_{2_{i}}, A_{3_{i}}\right)$, for $i=1, \ldots, n$ where $n$ is the number of weights related to the number of infected individuals, cost of preventive actions and treatment randomly and evaluate the fitness $\operatorname{SIR}\left(A_{1_{i}}, A_{2_{i}}, A_{3_{i}}\right)$, for $i=1, \ldots, n$.

Repeat

(2) Employed Bees Step. In the employed bees step, each employed bee generates solution as follows:

$A_{i}^{\prime}=\left(A_{1_{i}}^{\prime}, A_{2_{i}}^{\prime}, A_{3_{i}}^{\prime}\right)=\left\{\begin{aligned} A_{i}+\phi_{i}\left(A_{i}-A_{k}\right), & \text { if } r_{i}<M R \\ A_{i}, & \text { otherwise }\end{aligned}\right.$

where $\phi_{i}$ is a random number between $(-1,1), r_{i}$ is a random number between $(0,1)$, and $M R$ is the modification rate. Evaluate $\operatorname{SIR}\left(A_{1_{i}}^{\prime}, A_{2_{i}}^{\prime}, A_{3_{i}}^{\prime}\right)$, for $i=1, \ldots, n$. If new solution $\left(A_{1_{i}}^{\prime}, A_{2_{i}}^{\prime}, A_{3_{i}}^{\prime}\right)$ is better than $\left(A_{1_{i}}, A_{2_{i}}, A_{3_{i}}\right)$, replace $\left(A_{1_{i}}, A_{2_{i}}, A_{3_{i}}\right)$ with $\left(A_{1_{i}}^{\prime}, A_{2_{i}}^{\prime}, A_{3_{i}}^{\prime}\right)$.

(3) Onlooker Bees Step. Onlooker bees select one of solutions based on probability:

$$
p_{i}=\frac{\text { fitness }_{i}}{\sum_{i=1}^{n} \text { fitness }_{i}}, \quad i=1, \ldots, n
$$

Where in the minimization problem, the fitness can be obtained from:

$$
\text { fitness }_{i}=\left\{\begin{array}{cl}
\frac{1}{1+\operatorname{SIR}\left(A_{1_{i}}, A_{2_{i}}, A_{3_{i}}\right)}, & \text { if } \operatorname{SIR}\left(A_{1_{i}}, A_{2_{i}}, A_{3_{i}}\right) \geq 0 \\
1+\left|\operatorname{SIR}\left(A_{1_{i}}, A_{2_{i}}, A_{3_{i}}\right)\right|, & \text { if } \operatorname{SIR}\left(A_{1_{i}}, A_{2_{i}}, A_{3_{i}}\right)<0
\end{array}\right.
$$


(4) Scout Bees Step. Determine $\left(A_{1_{i}}, A_{2_{i}}, A_{3_{i}}\right)^{\min }$ and $\left(A_{1_{i}}, A_{2_{i}}, A_{3_{i}}\right)^{\max }$

$$
\begin{aligned}
& \left(A_{1_{i}}, A_{2_{i}}, A_{3_{i}}\right)^{\min }=\arg \min \left(\operatorname{SIR}\left(A_{1_{i}}, A_{2_{i}}, A_{3_{i}}\right), i=1 . . n\right) \\
& \left(A_{1_{i}}, A_{2_{i}}, A_{3_{i}}\right)^{\max }=\arg \max \left(\operatorname{SIR}\left(A_{1_{i}}, A_{2_{i}}, A_{3_{i}}\right), i=1 . . n\right)
\end{aligned}
$$

Determine the abandoned solution for the scout and replace it with:

$$
\begin{aligned}
& \left(A_{1_{i}}, A_{2_{i}}, A_{3_{i}}\right)=\left(A_{1_{i}}, A_{2_{i}}, A_{3_{i}}\right)^{\mathrm{min}}+ \\
& \quad \theta_{i}\left(\left(A_{1_{i}}, A_{2_{i}}, A_{3_{i}}\right)^{\mathrm{max}}-\left(A_{1_{i}}, A_{2_{i}}, A_{3_{i}}\right)^{\mathrm{min}}\right)
\end{aligned}
$$

where $\theta_{i} \sim U(0,1)$ then evaluate

$$
\operatorname{SIR}\left(A_{1_{i}}, A_{2_{i}}, A_{3_{i}}\right), \quad i=1, \ldots, n
$$

(5) Keep the best solution in the iteration.

\section{Simulation Results}

Parameters used on $\mathrm{ABC}$ simulations are:

1) The number of bees: 20

2) Modification rate: 0.8

3) Maximum iteration: 50

Parameters used on influenza model are :

1) Recruitment rate $\Lambda: 30$

2) Disease transmission rate $\beta: 0.05$

3) Natural death rate $\mu: 0.01$

4) Death by disease rate $\alpha: 0.03$

5) Recovery rate $\gamma: 0.7$

6) The lost immunity rate $\delta: 0.005$

The simulations of optimal control over the influenza model can be seen at Fig. 3-Fig. 7 while Fig. 2 is the ABC simulation.

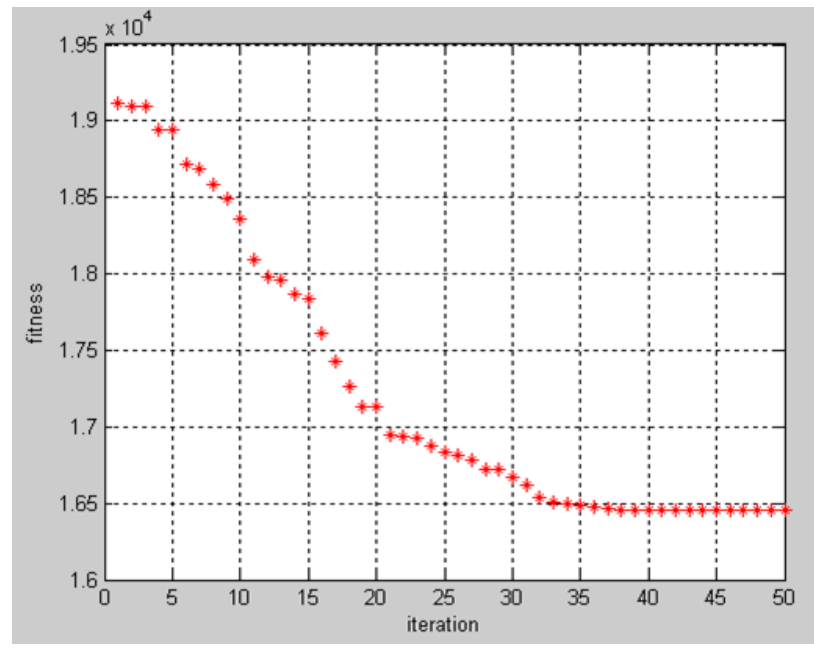

Fig. 2. Best fitness resulted by the $\mathrm{ABC}$ algorithm.

Figure 2 represents the first iteration, i.e. the bees choose the position of food source as weight randomly. At the optimization process, we update nectar information as fitness function so that bees choose the weight resulting in a minimum objective function.

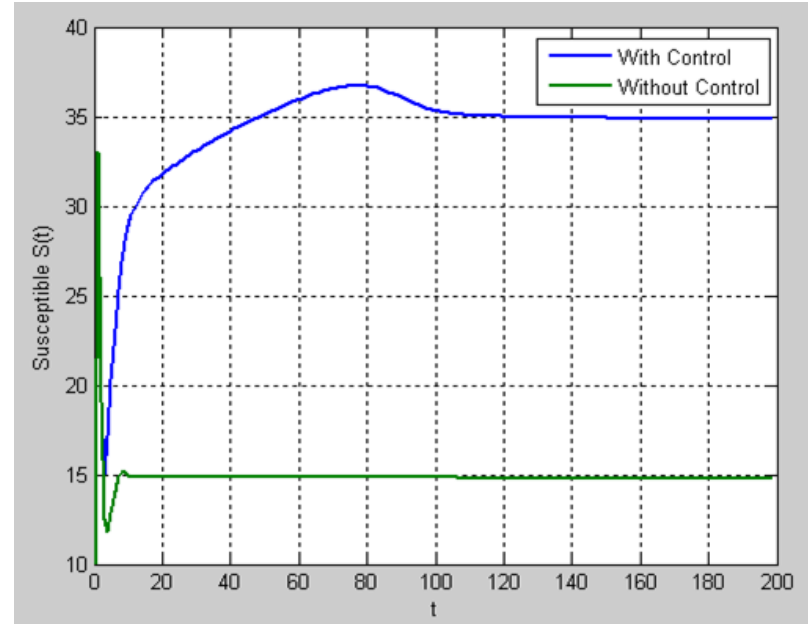

Fig. 3. Numerical solution of the number of susceptible individuals for two cases: with control and without control.

Figure 3 shows numerical solution of susceptible individuals for two cases, i.e. with control and without control. At the early time, the number of susceptible individuals with control is lower than without control because of the preventive action effect which decreases the number of susceptible individuals. However, as time increases, the number of susceptible individuals with control can be higher than without control because of the recruitment rate effect and the lost of immunity effect.

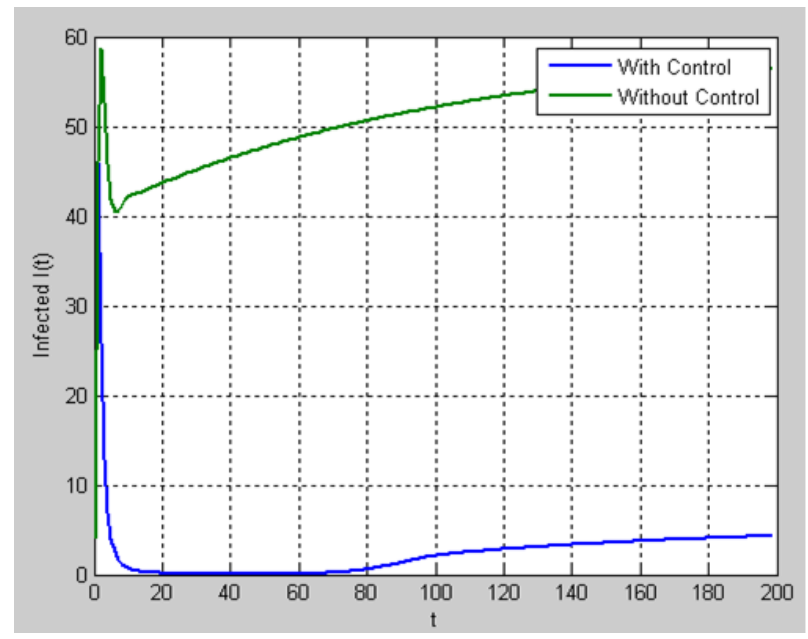

Fig. 4. Numerical solution of the number of infected individuals for two cases: with control and without control.

Figure 4 shows numerical solution of the number of infected individuals. The number of infected individuals with control is lower than without control because of the treatment effect which decreases the number of infected individuals. Figure 5 shows the numerical solution of the number of recovered individuals. The number of recovered individuals with control is higher than without control because of both prevention action and treatment effect which increase the number of recovered individuals. Figure 6 shows the control function of preventive actions. It shows that the preventive action must be applied massively since early time. Figure 7 shows the control 
function of treatment. It shows that there is a time interval where the treatment should not be applied massively.

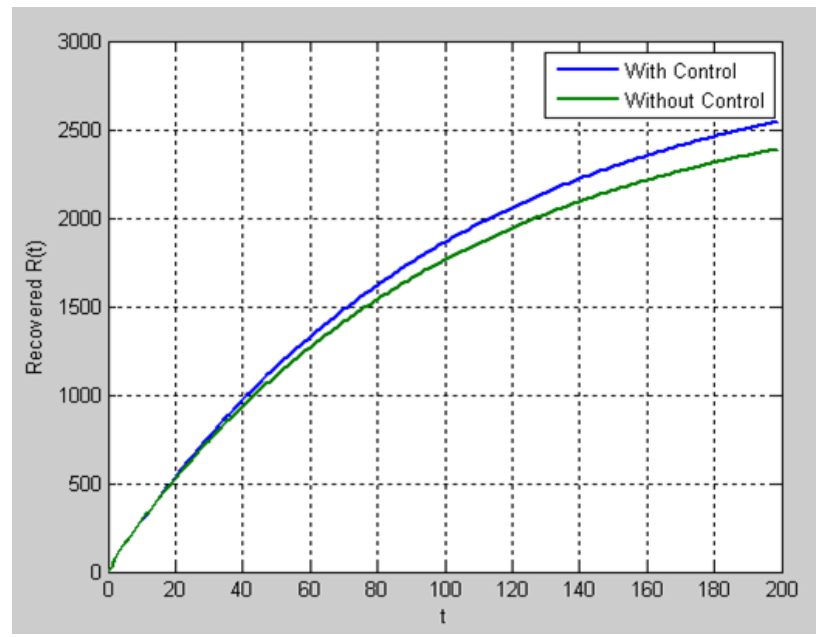

Fig. 5. Numerical solution of the number of recovered individuals for two cases: with control and without control.

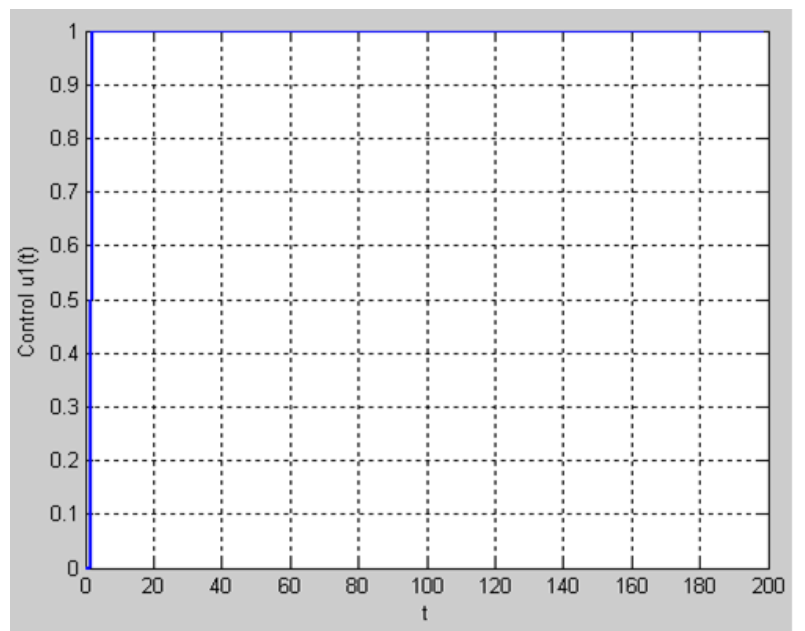

Fig. 6. Control function of preventive actions.

\section{CONCLUSION}

$\mathrm{ABC}$ method can select an optimal weight by using an optimal control of SIR epidemic model. From the simulation, initially the bees choose the position of food source as weight in a random fashion. At the optimization process, we update nectar information as fitness function so that bees choose the weight that results in minimum objective function. At the SIR simulation, based on parameters: recruitment rate, disease transmission rate, natural death rate, death by disease rate, recovery rate, disease mutation rate, can be seen from the comparison between the number of individuals for two cases: with and without control. Furthermore, we also obtain an optimal weight related to the number of infected individuals, cost of preventive actions and treatment.

\section{REFERENCES}

[1] “Influenza," http://en.wikipedia.org/wiki/influenza, accessed: 2017-07-01.

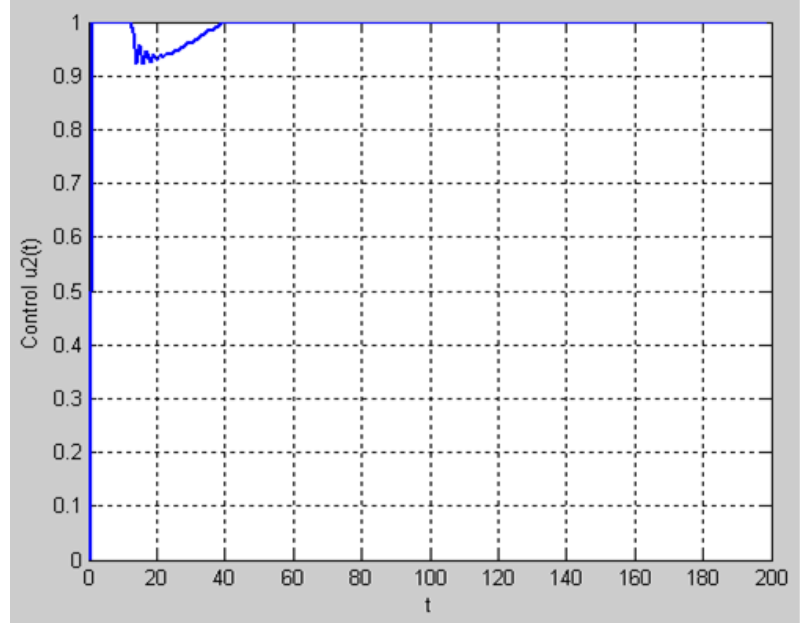

Fig. 7. Control function of treatment.

[2] M. Hia, O. Balatif, M. Rachik, and J. Bouyaghroumni, "Application of optimal control theory to an seir model with immigration of infectives," IJCSI International Journal of Computer Science Issues, 2013.

[3] E. Bakare, A. Nwagwo, and E. Danso-Addo, "Optimal control analysis of an sir epidemic model with constant recruitment," International Journal of Applied Mathematical Research, vol. 3, no. 3, pp. 273-285, 2014.

[4] Z. Michalewicz, C. Janikow, and J. Krawczyk, "A modified genetic algorithm for optimal control problems," Computers \& Mathematics with Applications, vol. 23, no. 12, pp. 83-94, 1992.

[5] D. Karaboga and B. Basturk, "A powerful and efficient algorithm for numerical function optimization: artificial bee colony (abc) algorithm," Journal of global optimization, vol. 39, no. 3, pp. 459-471, 2007.

[6] J. Burl, Linear optimal control: H (2) and H (Infinity) methods. AddisonWesley Longman Publishing Co., Inc., 1998.

[7] D. Rahmalia and T. Herlambang, "Application ant colony optimization on weight selection of optimal control seir epidemic model," in Proceeding the 7th Annual Basic Science International Conference, Dec. 2017, pp. 196-199.

[8] T. Liao, D. Aydın, and T. Stützle, "Artificial bee colonies for continuous optimization: Experimental analysis and improvements," Swarm Intelligence, vol. 7, no. 4, pp. 327-356, 2013.

[9] O. Sharomi and T. Malik, "Optimal control in epidemiology," Annals of Operations Research, vol. 251, no. 1-2, pp. 55-71, 2017. 